\title{
Research on the Online Factors of Second-Hand Houses that Affect Consumers' Off-Line Viewing
}

\author{
Yun Tang ${ }^{1, a, ~}{ }^{*}$, Fu He ${ }^{2, b}$, Ling $\mathrm{Li}^{3, c}$, Huaxue Zhuang ${ }^{4, d}$ \\ ${ }^{1}$ Department of electronic commerce, sichuan agricultural university, chengdu, sichuan, China \\ ${ }^{2}$ Department of electronic commerce, sichuan agricultural university, chengdu, sichuan, China \\ ${ }^{3}$ Department of electronic commerce, sichuan agricultural university, chengdu, sichuan, China \\ ${ }^{4}$ Department of marketing, sichuan agricultural university, chengdu, sichuan, China

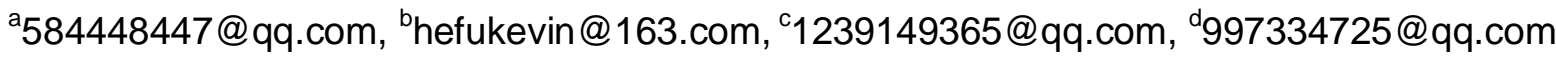 \\ *Yun Tang
}

Keywords: Online attention, Distance to subway, Length of construction time, Linear regression, Behavioral decision theory

\begin{abstract}
Using linear regression model on the combination of behavioral decision theory to analyse the data of second-hand housing in 2505 Beijing districts and 2343 Chengdu districts, explore the amount of second-hand housing online attention, the distance from the subway and build the length of time for consumers offline check the influence of second-hand housing, the study shows that the secondary line of concern, the distance from the subway and build the length of time will have a significant impact on consumers' choice of offline viewing, based on marketing for real estate agents website of second-hand housing to make the corresponding guidance.
\end{abstract}

\section{影响消费者线下看房的二手房线上因素研究}

\author{
汤韵 ${ }^{1, a}$, 何甫 ${ }^{2, b}$, 李玲 $3, c$, 庄华雪 $4, d$ \\ ${ }^{1}$ 四川农业大学电子商务系, 都江堰, 成都, 四川, 中国 \\ ${ }^{2}$ 四川农业大学电子商务系, 都江堰, 成都, 四川, 中国 \\ ${ }^{3}$ 四川农业大学电子商务系, 都江堰, 成都, 四川, 中国 \\ ${ }^{4}$ 四川农业大学市场营销系, 都江堰, 成都, 四川, 中国 \\ a584448447@qq.com, bhefukevin@163.com, c1239149365@qq.com, d997334725@qq.com \\ *汤韵
}

关键词: 线上关注量; 到地铁距离; 建造时间长短; 线性回归; 行为决策理论

摘要.利用线性回归模型结合行为决策理论来对链家上 2505 条北京地区和 2343 条成都地区的 二手房数据进行分析，探索在线二手房的线上关注量、到地铁距离以及建造时间长短对消费 者线下查看二手房的影响, 研究显示二手房的线上关注量、到地铁距离以及建造时间长短都 会对消费者选择线下看房有着显著的影响, 以此为依据为房产中介网站的二手房营销做出相 应的指导。

\section{1.引言}

住宅消费是我国国民经济的一个不可或缺的部分，在我国的国民经济运行中有着举足轻 
重的作用 ${ }^{[1]}$; 同时, 住宅消费需求的增长是经济可持续发展的保证 ${ }^{[2]}$; 此外, 购房不仅仅是市 场经济行为, 同时也是文化融合重构的结果 ${ }^{[3]}$ 。近年来, 国内许多一线城市和部分二线城市 出现了中心城区用地日益稀少的现象，土地资源的稀缺性导致二手房正在逐步取代一手房。 在过高的商品房价、国家限购等宏观政策以及消费者越来越理性选择住房思想等的影响下， 具有明显价格优势的二手房市场开始逐渐兴起。二手房市场作为我国房地产市场一个重要组 成部分，对于二手房购买行为的研究，能给房地产市场一些有益的指导。

目前国内外很多学者都对影响二手房价格的因素作了研究，陈瑛 ${ }^{[4]}$ 等通过回归分析表示 二手房价格受地理区位影响大; 刘丽 ${ }^{[5]}$ 用协整方法得出人均可支配收入、地区 GDP、一手房

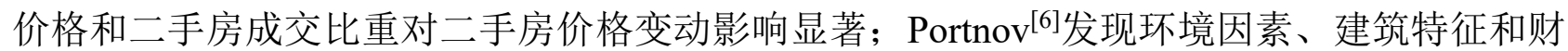
产价值三组变量都可以影响二手房的市场价值; $\operatorname{Han}^{[7]}$ 等人发现价格风险对家庭购买的时间和 规模的负面影响, 但对具有较强对冲动机的家庭产生积极影响。在二手房消费方面, 影响消 费者购买的因素有很多，章霞 ${ }^{[8]}$ 等通过调查问卷实证分析指出国家政策、外部交通、二手房 中介是高价房下市民二手房消费的影响因素; 郑云坚 ${ }^{[9]}$ 通过门限回归分析指出城市轨道交通 对二手房消费的影响; 张清源 ${ }^{[10]}$ 利用回归分析得出住房面积越小、朝南、满五唯一的住房单 价更高, 学区房、与市中心天安门越近的二手房越受欢迎。这些对二手房线下因素的探索对 我国二手房的相关研究提供了很好的方向。但这些研究大部分集中于二手房的线下因素研究, 很少涉及到线上因素。

目前, 我互联网发展十分迅速。根据 CNNIC 在 2017 年 6 月发布的第 40 次《中国互联网 络发展状况统计报告》显示, 截至 2017 年 6 月, 中国网民规模达到 7.51 亿, 占全球网民总 数的五分之一, 中国的互联网普及率为 $54.3 \%$ 。这也不断推动着我国网络营销的发展, 许多 在线二手房中介平台也随之诞生, 不断显示出在线信息渠道的重要性 ${ }^{[11]}$, 了解在线用户的态 度和行为也就十分必要。因此因而我国二手房行业应该将电子商务和传统的营销方式相结合 [12]。在网站的“1+4”模式下[13]，探索在线二手房的市场现状及前景、影响消费者购买二手房 的因素以及如何设计房地产网站建设与发展的主要战略方向, 都值得我们深入的研究和探讨。

根据 CNNIC 发布的《2016 年中国网民搜索行为调查报告》显示，目前我国综合搜索引 擎用户规模达 6.02 亿，使用率为 $82.4 \%$ 。消费者也越来越依赖于网络进行信息搜素。而现在 大部分二手房中介商都会在线上展示房源信息, 但是由于目前信息泛滥, 信息搜素城本较高, 导致在线信息得不到很好的利用, 所以如何利用已有信息进行二手房的线上营销就显得尤为 重要。本文就通过研究线上展示的二手房信息对消费者线下的看房行为影响, 通过搜集整理 线上的二手房信息，建立线性回归模型来研究线上信息对消费者的影响。

\section{2.相关理论及假设}

通过查阅整理大量文献发现，目前我国学者对二手房市场的研究主要是在影响其价格因 素的分析方面, 而对影响消费者二手房购买行为研究较少。其次, 互联网信息资源是目前了 购房者获取信息的主要途径, 现在打分的二手房信息都通过互联网进行展示, 对消费者购房 决策起着重要作用, 因此研究线上信息对消费者购房选择行为是很有必要的, 而在研究消费 者购买二手房行为方面很少有将线上线下相结合进行探索的。因此，本文将结合已有的相关 文献，在分析二手房购买行为影响因素之基础上，从线上关注量、二手房的建成时间以及二 手房距离地铁距离的角度出发, 同时考虑二手房的面积大小、价格和户型等因素, 研究影响 消费者查询购买二手房的线上因素。

\section{1 行为决策理论}

行为决策理论的起步始于阿莱斯悖论和爱德华兹悖论 ${ }^{[14]}$ 的提出, 是针对理性决策理论难 以解决的问题另辟蹊径发展起来的。1961 年, Edwards ${ }^{[15]}$ 教授提出了“决策权重”的思想, 被 后世广泛用于消费者行为分析。该理论研究同样具有三大特点 ${ }^{[16]}$ : (1)出发点是决策者的决策 
行为; (2)研究集中在决策者的认知和主观心理过程, 关注决策行为背后的心理解释, 而不是 对决策正误的评价; (3)从认知心理学的角度, 研究决策者在判断和选择中信息的处理机制及 其所受的内外部环境的影响, 进而提炼出理性决策理论所没有考虑到的行为变量, 修正和完 善理性决策模型。现目前决策行为理论已经广泛运用在股市 ${ }^{[17]}$ 、营销 ${ }^{[18]}$ 和管理 ${ }^{[19]}$ 方面。

当消费者产生购买二手房的需求之后，就会在看房之前大量搜集二手房信息以支持自己 的决策，而这些信息处理最基本的面积价格等，还会考虑二手房的线上受欢迎程度、交通情 况和二手房的新旧情况，因而使用行为决策理论可以较好的研究消费者线下带看行为的影响 因素。

\section{2 线上关注量}

在线二手房房源的价格、面积和户型等基本信息在二手房线上营销中有着至关重要的作 用, 能够一定程度上反映房源的质量等信息, 是消费者了解房源的基础信息。但是这些信息 不能反映出在线二手房的受欢迎程度。因此, 链家网站往往在二手房的详情页展示二手房的 地铁、公交车等交通信息、交易属性信息，此外还会展示出房源的关注量等系统生成信息。 系统生成信息 ${ }^{[20]}$ 是指由在线系统根据用户的浏览行为和数据所产生的信息,这部分信息在网 络营销和电子商务领域中有着至关重要的作用。例如: 在体验型产品中,产品评论次数和销量

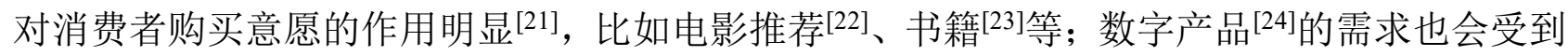
网络效应的影响, 例如在线音乐 ${ }^{[25]}$ 等。因此消费者在线上选择二手房时候更加需要这一部分 系统生成信息来进行决策。

在线二手房的关注量是众多消费者关注该二手房之后生成的，可以间接反映出该二手房 的受欢迎程度, 现在已经有研究指出在线评论 ${ }^{[26]}$ 会对商品是否受欢迎有着显著影响。房源的 关注量是由消费者已有的行为产生, 同样其可信度也就更高。因此提出假设:

H1：在线二手房已有的关注量会对消费者线下看房次数有显著的正向影响。

\section{3 二手房的新旧程度和地铁距离}

二手房营销不仅受到线上关注量的影响，还会受到线下诸多因素的影响，该区域的交通 [27]、人口 ${ }^{[28]}$ 与金融环境 ${ }^{[29]}$ 都深刻的影响着该地区的房价。这经济发达地区往往交通便捷, 地 铁站口较多, 方便人们出行。但此地区的房价也相应较高, 大部分往往是具有一定经济实力 的人才能购买。所以虽然交通方便, 但是受欢迎程度较低; 相反那些距离地铁较远, 房价便 宜的二手房更受欢迎, 因此提出假设:

$\mathrm{H} 2$ : 二手房距离地铁远对消费者线下看房次数具有显著正向影响

建造时间因素显著影响二手房价格 ${ }^{[30]}$, 而建造时间一定程度上可以决定房屋的新旧程度。 但是目前大部分研究都只是考虑地铁距离和建造时间对二手房价格的影响, 很少考虑对消费 者购买决策行为的影响, 所以提出假设:

H3：建造的时间间隔较长对消费者线下看房次数有显著的负向影响

\section{3.实证分析}

\section{1 数据和变量描述}

此次实验的数据来源于链家，链家是一家房产交易的服务平台，链家网站会提供二手房 房源较为全面的信息, 包括房源的基本属性和交易属性、房源特色、关注量和带看次数等信 息。因此我们选择链家网进行数据的收集。我们通过一个基于 Python 的爬虫程序进行线上的 数据收集。全国各大省市中, 北上广深等一线城市的房地产发展迅速, 二手房交易规模较大, 同时成都、武汉等新一线城市也不断崛起。为了研究不同城市的数据, 因此我们选择了北京 和成都两个城市进行数据的收集。除去缺失值较多的部分数据后, 最终得到了 2505 条北京地 区二手房数据和 2343 条成都地区的二手房数据，两地区共 4848 条数据。 
表格 1 数据特征

\begin{tabular}{lcccccc}
\hline \multicolumn{1}{c}{ 变量 } & 观测值 & 平均值 & 标准差 & 最小值 & 最大值 & VIF \\
\hline Ln(times) & 4,848 & 2.85 & 1.44 & 0 & 6.259 & -- \\
Ln(attention) & 4,848 & 3.51 & 1.26 & 0 & 7.04 & 1.53 \\
Ln(subway) & 4,848 & 9.81 & 3.75 & 1 & 13 & 1.09 \\
Ln(interval) & 4,848 & 2.37 & 0.68 & 0 & 7.61 & 1.36 \\
Ln(square) & 4,848 & 4.55 & 0.40 & 3.11 & 5.89 & 3.44 \\
Ln(price) & 4,848 & 5.76 & 0.76 & 3.14 & 8.1 & 1.71 \\
Ln(room) & 4,848 & 2.50 & 0.89 & 1 & 9 & 2.69 \\
Ln(date) & 4,848 & 2.50 & 2.41 & 1 & 13 & 1.42 \\
\hline
\end{tabular}

表格 2 变量的相关性检验

\begin{tabular}{lllllllll}
\hline 变量 & \multicolumn{1}{c}{ Times } & Attention & Subway & Interval & Square & Price & Room & Date \\
\hline Times & 1.0000 & & & & & & & \\
Attention & $0.7451^{*}$ & 1.0000 & & & & & & \\
Subway & $0.3176^{*}$ & $0.1853^{*}$ & 1.0000 & & & & & \\
Interval & $0.1424^{*}$ & $0.2469^{*}$ & $-0.0965^{*}$ & 1.0000 & & & & \\
Square & $-0.0615^{*}$ & $-0.1049^{*}$ & $-0.0314^{*}$ & $-0.1519^{*}$ & 1.0000 & & & \\
Price & $0.2709^{*}$ & $0.2937^{*}$ & -0.0258 & $0.3735^{*}$ & $0.3790^{*}$ & 1.0000 & & \\
Room & $-0.1047^{*}$ & $-0.0961^{*}$ & -0.0066 & $-0.1137^{*}$ & $0.7831^{*}$ & $0.2004^{*}$ & 1.0000 & \\
Date & $0.5007^{*}$ & $0.4714^{*}$ & $0.2085^{*}$ & $0.0726^{*}$ & $0.1882^{*}$ & $0.2571^{*}$ & $0.1294^{*}$ & 1.0000 \\
\hline
\end{tabular}

*表示显著水平超过 $5 \%$

表格 3 变量描述

\begin{tabular}{llll}
\hline 变量类别 & 变量名称 & 变量描述 & 测量方式 \\
\hline 自变量 & 带看次数 & 消费者线下看房次数 & 线下带看次数 \\
自变量 & 线上关注量 & 网站上关注该房源的人数 & 线上关注该房源的人数 \\
& 地铁距离 & 该房源到最近地铁站距离 & 距离的长短 \\
& 时间间隔 & 建成时间到目前时间长短 & 目前年份减去建成年份 \\
控制变量 & 大小 & 面积大小 & 面积 \\
& 价格 & 房源的总价 & 房源的总价 \\
& 户型 & 卧室数目 & 卧室的数量 \\
& 时间 & 发布时间 & 现在的月份减去发布的月份 \\
\hline
\end{tabular}

\section{2 模型估计}

消费者线下带看次数的变化往往受到多个因素的影响，因此使用多元线性回归模型来检 验线上关注量、到地铁距离以及建造时间长短对消费者线下带看次数影响。在此次研究中, 以二手房的线下带看次数作为因变量, 用来反映二手房的转换程度; 自变量为该二手房的线 上关注量、到地铁距离以及建造时间长短，而二手房的大小、价格、户型和发布时间等作为 控制变量纳入模型进行分析。为了避免数据间的多重共线性同时便于分析, 所以所有的数据 我们取对数纳入模型计算。

$$
\begin{gathered}
\operatorname{Ln}(\text { times })=\beta 0+\beta 1 \operatorname{Ln}(\text { room })+\beta \operatorname{Ln}(2 \text { date })+\beta 3 \operatorname{Ln}(\text { square })+\beta 4 \operatorname{Ln}(\text { price })+\beta 5 \operatorname{Ln}(\text { attention }) \\
+\beta 6 \operatorname{Ln}(\text { interval })+\beta 7 \operatorname{Ln}(\text { subway })
\end{gathered}
$$

times 表示该房源消费者的线下被带看次数，可反映线上向线下转化的程度; room 表示 的该房源的卧室数，用反映该二手房的户型; date 表示的该二手房在线上发布时间的长短, 分别用到 13 表示，1 表示发布时间在一个月以内，2 表示发布时间超过一个月但是不超过两 个月以此类推; square 表示的是该房源的面积; price 表示该二手房的总价格; attention 表示 的是该二手房在线上的关注量的多少; interval 表示的建成时间距离现在时间的长短; subway 表示的是该二手房距离最近地铁站的距离, 分别用 1 到 13 表示, 在一千米以内用 1 表示, 在 一千米以外两千米以内用 2 表示，以此类推。这些数据均在链家二手房的详情页有展示。 


\section{4. 研究结论}

由于回归模型中可能存在变量间相关等问题，本文在回归分析采用逐步回归的方式来检 验模型可能存在的多重共线性问题。经过 Stata 统计软件的分析。回归模型通在线二手房的线 上关注量、到地铁距离以及建造时间长短的计算, 最终 $R^{2}$ 和 $\mathrm{t}$ 检验都符合要求, 具体结果如 下图所示:

表格 4 实证结果

\begin{tabular}{lcc}
\hline 变量 & 模型 1 & 模型 2 \\
\hline 常数项 & $3.01 * * *$ & $-1.057^{* * *}$ \\
& $(11.44)$ & $(-4.78)$ \\
Ln(attention) & & $0.701^{* * *}$ \\
& & $(56.05)$ \\
Ln(subway) & $0.063^{* * *}$ \\
& & $(18.02)$ \\
Ln(interval) & $-0.949 * * *$ \\
& & $(-4.31)$ \\
Ln(square) & $-0.685^{* * *}$ & -0.718 \\
& $(-9.22)$ & $(-1.20)$ \\
Ln(price) & $0.434^{* * *}$ & $0.153^{* * *}$ \\
& $(17.45)$ & $(7.02)$ \\
Ln(room) & $-0.107^{* * *}$ & $-0.166^{* * *}$ \\
& $(-3.44)$ & $(-7.10)$ \\
Ln(date) & $0.290^{* * *}$ & $0.101 * * *$ \\
& $(39.50)$ & $(15.96)$ \\
Adjust-R & 0.324 & 0.620 \\
$\mathrm{~F}$ & 580.56 & 1128.39 \\
$\mathrm{~N}$ & 4,848 & 4,848 \\
\hline ***表示显著水平为 $1 \%, * *$ 表示显著水平为 $5 \%, *$ 表示显著水平为 $10 \%$
\end{tabular}

如表 3 所示, 综合两地区的关注量（ $\mathrm{B}=0.701, \mathrm{~T}=56.05, \mathrm{P}<0.01 ）$ 的回归系数显著, $\mathrm{H} 1$ 得 到验证, 即在线二手房已有的关注量会对消费者线下看房次数有显著的正向影响。同时综合 两地区二手房到地铁距离 $(\mathrm{B}=0.063, \mathrm{~T}=18.02, \mathrm{P}<0.01)$ 的回归系数也十分显著, 验证了假设 $\mathrm{H} 2$, 即二手房距离地铁远对消费者线下看房次数具有显著正向影响。而时间间隔的系数

$(\mathrm{B}=-0.949, \mathrm{~T}=-1.31, \mathrm{P}<0.01)$ 也是显著的, 这也就说明建造的时间间隔较长对消费者线下看房 次数有显著的负向影响。此外, 在结果当中二手房的价格, 户型等信息对消费者线下看房次 数具有负向影响。

\section{5.讨论与建议}

本文在相关文献分析整理的基础上，从在线二手房的线上展示的信息出发出发，整合线 上线下信息对二手房的带看次数影响进行研究。并着重研究已有的在线二手房的线上关注量、 到地铁距离以及建造时间长短对线上二手房营销过程中的效果。同时本文结合房屋自身面积、 价格等属性, 提出解释线上二手房线下带看次数的假设模型, 发现了二手房的线上关注量、 到地铁距离会对二手房的线上营销产生重要的影响。这也就表明, 购买者更愿意选择那些关 注量较大以及原理地铁的二手房, 因此房产中介商在进行网络营销的时候要抓住消费者的这 种心理, 将诸如以往消费者行为类信息能够放在突出位置, 这样能够很好的吸引消费者的线 上关注，从而才能进一步转化为线下的成交。

由于两地区的数据可能存在相互干扰的情况, 同时也是为了研究不同地区的影响效果, 因而分别度两地区的数据建立线性回归模型, 结果如下所示: 
表格 5 各地区的分析结果

\begin{tabular}{|c|c|c|c|c|}
\hline \multirow[t]{2}{*}{ 变量 } & \multicolumn{2}{|c|}{ 北京 } & \multicolumn{2}{|c|}{ 成都 } \\
\hline & 模型 1 & 模型 2 & 模型 1 & 模型 2 \\
\hline 常数项 & $\begin{array}{l}3.811 * * * \\
(11.56)\end{array}$ & $\begin{array}{l}-0.670^{* * *} \\
(-2.98)\end{array}$ & $\begin{array}{l}3.813^{* * *} \\
(8.51)\end{array}$ & $\begin{array}{l}-0.695^{*} \\
(-1.81)\end{array}$ \\
\hline $\operatorname{Ln}($ attention) & & $\begin{array}{l}0.283 * * * \\
(19.38)\end{array}$ & & $\begin{array}{l}0.780 * * * \\
(39.99)\end{array}$ \\
\hline Ln(subway) & & $\begin{array}{l}0.249^{* * *} \\
(61.30)\end{array}$ & & $\begin{array}{l}0.271 * * * \\
(60.13)\end{array}$ \\
\hline Ln(interval) & & $\begin{array}{l}-0.046^{* *} \\
(-2.23)\end{array}$ & & $\begin{array}{l}-0.065^{*} \\
(-1.82)\end{array}$ \\
\hline Ln(square) & $\begin{array}{l}-0.269 * * * \\
(-2.99)\end{array}$ & $\begin{array}{l}-0.056 \\
(-0.19)\end{array}$ & $\begin{array}{l}-0.362^{* *} \\
(-2.41)\end{array}$ & $\begin{array}{l}-0.267 * * \\
(-2.28)\end{array}$ \\
\hline Ln(price) & $\begin{array}{l}0.425 \\
(0.75)\end{array}$ & $\begin{array}{l}0.125^{* * * *} \\
(4.20)\end{array}$ & $\begin{array}{l}-0.094 \\
(-1.06)\end{array}$ & $\begin{array}{l}0.431 * * * \\
(5.89)\end{array}$ \\
\hline Ln(room) & $\begin{array}{l}-0.104^{* * *} \\
(-2.72)\end{array}$ & $\begin{array}{l}-0.70 * * * \\
(-3.30)\end{array}$ & $\begin{array}{l}-0.024 \\
(-0.49)\end{array}$ & $\begin{array}{l}-0.153^{* * *} \\
(-3.97)\end{array}$ \\
\hline Ln(date) & $\begin{array}{l}0.244 * * * \\
(30.63)\end{array}$ & $\begin{array}{l}0.047 * * * \\
(2.23)\end{array}$ & $\begin{array}{l}0.352^{* * * *} \\
(26.90)\end{array}$ & $\begin{array}{l}0.102 * * \\
(8.59)\end{array}$ \\
\hline Adjust-R & 0.275 & 0.811 & 0.235 & 0.547 \\
\hline $\mathrm{F}$ & 238.03 & 1542.67 & 181.01 & 405.51 \\
\hline $\mathrm{N}$ & 2505 & 2505 & 2343 & 2343 \\
\hline
\end{tabular}

除了两个地区的综合结果，通过上表还可以看出，成都北京两个地区中，在线二手房的 线上关注量、到地铁距离以及建造时间长短对二手房的线下带看次数也是有显著影响的。北 京地区的关注量 $(\mathrm{B}=0.283, \mathrm{~T}=19.38, \mathrm{P}<0.01)$ 和成都地区的线上关注量 $(\mathrm{B}=0.780, \mathrm{~T}=39.99, \mathrm{P}<0.01)$ 系数均显著, 也表明了在线二手房已有的关注量会对消费者线下看房次数有显著的正向影响; 而在二手房到地铁, 北京地区 $(\mathrm{B}=0.249, \mathrm{~T}=61.30, \mathrm{P}<0.01)$ 和成都地区 $(\mathrm{B}=0.271, \mathrm{~T}=6.13, \mathrm{P}<0.01)$ 系数也是显著的, 也验证了假设 $\mathrm{H} 2$, 即即二手房距离地铁远对消费者线下看房次数具有显著 正向影响; 最后两个地区房屋建造的时间间隔, 北京地区（ $\mathrm{B}=-0.046, \mathrm{~T}=-2.23, \mathrm{P}<0.05)$ 和成都 地区（ $\mathrm{B}=-0.065, \mathrm{~T}=-1.82, \mathrm{P}<0.10 ）$ 也是显著的。综上结果表明，在线二手房的线上关注量、到 地铁距离以及建造时间长短这一类的展示信息都会对消费者线下的带看次数产生显著影响。

\section{6.结语}

本文从已有的研究出发，探索了在线的二手房信息对消费者线下带看次数的影响，针对 已有文章大部分研究二手房房价影响因素的问题, 我们通过建立了多元线性回归模型, 实证 研究验证了二手房的线上关注量、到地铁距离会对消费者在线选择二手房的产生显著影响, 同时也是对新房和租房线上营销的合理探索。同时本文也存在着一些不足，例如二手房的信 息不仅包括文字信息，同时也包括了图片信息，这些图片也会很大程度上影响消费者的选择; 而且这次的数据全部取样于链家网站, 也可以去其他的在线二手房销售网站进行对比, 这样 结果也就更为准确。

\section{References}

[1] Crowe C, Dell'Ariccia G, Igan D, et al. How to deal with real estate booms: Lessons from country experiences[J]. Journal of Financial Stability, 2013, 9(3):300-319.

[2] Jie Zheng. Importance and strategic advice for starting residential consumption [J]. China Real Estate Finance, 2000(2):3-6.

[3] XinRu Xiao. Analysis on the Willingness of Young Floating Population to Purchase Houses in Big Cities and Its Influencing Factors_-Taking Shanghai as an Example [J]. Shanghai 
Economic Research, 2014(8):121-128.

[4] Ying Chen, Yanfei Pei, Hui Li. Study on the Difference Law and Influence Factors of Housing Price Gradient in Xi'an City [J]. Statistics and Information Forum, 2008, 23(12):37-42.

[5] Li Liu, Aisong Li. Research on the Factors Affecting the Price Change of Second-hand Housing in Guangzhou [J]. Price theory and practice, 2008(5):42-43.

[6] Portnov B A, Odish Y, Fleishman L. Factors Affecting Housing Modifications and Housing Pricing: A Case Study of Four Residential Neighborhoods in Haifa, Israel[J]. Journal of Real Estate Research, 2005, 27(4):371-408.

[7] Han L. The Effects of Price Risk on Housing Demand: Empirical Evidence from U.S. Markets[J]. Review of Financial Studies, 2010, 23(11):3889-3928.

[8] Xia Zhang, Rongqing Chen, Yafei Wang. Investigation on the Factors Affecting the Consumption Behavior of Second-hand Houses under the High Housing Rate-Taking Chengdu as an Example [J]. Economic research guide, 2012(4):161-163.

[9] Yunjian Zheng, Xirong Chen, Zeyu Wang. The Influence of Urban Rail Transit Accessibility on the Price of Second-hand House-An Empirical Analysis Based on Threshold Model [J]. Fujian Forum (Humanities and Social Sciences Edition), 2017(6):27-32.

[10]Qingyuan Zhang, Guochan Su, Ruobing Liang. Who is affecting the transaction price of second-hand housing - empirical evidence from Beijing [J]. Finance and trade economy, 2017, 38(2):126-140.

[11]Kim D Y, Lehto X Y, Morrison A M. Gender differences in online travel information search: implications for marketing communications on the internet. [J]. Tourism Management, 2007, 28(2):423-433.

[12]Dejian Li. The use of e-commerce in the real estate intermediary industry [J]. Journal of Guizhou University of Finance and Economics, 2001(6):81-85.

[13]Faliang Zhong, Changchun Feng. Analysis of the Status Quo and Development Direction of Real Estate Websites in Mainland China [J]. Civil and Environmental Engineering, 2001, 23(z1):89-95.

[14] SCOTTPLOUS. Decision and judgment [M]. People Post Press, 2004.

[15]Edwards W. Behavioral decision theory. [M]. Cambridge University Press, 1994.

[16]Cheng Huang. Behavior Decision Theory and Empirical Research Methods of Decision Behavior [J]. Economic latitude, 2006, 2006(5):102-105.

[17]Cheng Huang, Jianmei Yang. Experimental Study on Inferred Behavior of Short-Term Price Trends of Chinese Stock Market Investors [J]. Journal of Hunan University (Social Science Edition), 2006, 20(3):74-81.

[18] John L K. The consumer psychology of online privacy: insight and opportunities from behavioral decision theory[J]. 2016: págs. 619-646.

[19]Quan Chen, Kaili Zhang, Guohong Shi. esearch on the Strategic Decision Process of Executive Team: A Theoretical Model_—Based on Behavioral Decision Theory [J]. Management modernization, 2014(1):36-38.

[20] Westerman D, Spence P R, Heide B V D. A social network as information: The effect of system generated reports of connectedness on credibility on Twitter[J]. Computers in Human Behavior, 2012, 28(1):199-206. 
[21]Zongwei Li, Yanhui Zhang, Dongqing Luan. What factors influence consumers' online purchase decisions? - The driving role of customer perceived value [J]. Management review, 2017, 29(8):136-146.

[22] Amolochitis E, Christou I T, Tan Z H. Implementing a Commercial-Strength Parallel Hybrid Movie Recommendation Engine[J]. IEEE Intelligent Systems, 2014, 29(2):92-96.

[23] Chevalier J A, Mayzlin D. The Effect of Word of Mouth on Sales: Online Book Reviews[J]. Social Science Electronic Publishing, 2006, 43(3):345-354.

[24]Xinli Shang, Yunfei Ma. Analysis of Factors Affecting Supply and Demand of Digital Products [J]. Library science research, 2014(10):55-58.

[25] Dewan S, Ramaprasad J. Research Note---Music Blogging, Online Sampling, and the Long Tail[J]. Information Systems Research, 2012, 23(3-Part-2):1056-1067.

[26] Yingzhi Pan, Jinhong Cui, Huan Wang. An Empirical Study of the Impact of Online Comments on the Sales Revenue of Different Popularity Experience Products [J]. Library and information work, 2011, 55(24):126-131.

[27] Yong Xiang, Yujing Yang, Xiaolan Shu. Analysis of the Influence of Urban Traffic on House Price Based on Characteristic Price [J]. Technical Economics and Management Research, 2009(6):81-83.

[28] Jianwei Xu, Qiyuan Xu, Fan He. The demographic factors behind the rise in housing prices: international experience and Chinese evidence [J]. world economy, 2012(1):24-42.

[29]Huayi Yu, Dong Chen. Research on the correlation between land price, interest rate and house price in China [J]. Economic review, 2009(4):41-49.

[30]Hongjun Zhao, Yi Peng. The Non-linear Influence of Location Factors on the Price of Second-hand Houses: A Case Study of Shanghai [J]. Urban problem, 2017(7):60-66. 